Nervenarzt 2021 · 92:501-506

https://doi.org/10.1007/s00115-020-01002-y

Angenommen: 7. September 2020

Online publiziert: 1 . Oktober 2020

(c) Springer Medizin Verlag GmbH, ein Teil von Springer Nature 2020

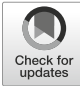

Kristina Adorjan ${ }^{1,2} \cdot$ Dorothea Gaudernack ${ }^{3}$ Julia Beer ${ }^{3}$.

Christine von Massenbach ${ }^{4}$. Peter Falkai' · Thomas Pollmächer ${ }^{5}$. Oliver Pogarell

'Klinik für Psychiatrie und Psychotherapie, LMU-Klinikum, München, Deutschland

${ }^{2}$ Institut für Psychiatrische Phänomik und Genomik (IPPG), LMU-Klinikum, München, Deutschland

${ }^{3}$ Amt für öffentlich-rechtliche Unterbringung (AfoerU), Nördlingen, Deutschland

${ }^{4}$ Bayerisches Staatsministerium der Justiz (StMJ), München, Deutschland

${ }^{5}$ Zentrum für psychische Gesundheit, Klinikum Ingolstadt, Ingolstadt, Deutschland

\title{
Zwangsmaßnahmen und die SARS-CoV-2-Pandemie
}

Rücksprache mit dem Konsiliardienst aufgrund der psychischen Erkrankung (Alkoholintoxikation) eine Verlegung in die Klinik für Psychiatrie und Psychotherapie (dortige COVID-Station), womit der Patient einverstanden war.

Dennoch blieb er auch nach Verlegung wenig compliant, drängte immer wieder auf Entlassung und war erst dann zumindest teilweise kooperativ, nachdem ihm das Rauchen im Patientenzimmer gestattet wurde. Letzteres erfolgte vor dem Hintergrund des COVID-19-Verdachts. Es sollte so ein eigenmächtiges Verlassen des Isolierzimmers bzw. der Station insgesamt zumindest bis zum Abschluss der Diagnostik bzw. rechtlichen Klärung der Situation vermieden werden. Dabei sah sich das medizinische Personal sowohl in der Notaufnahme als auch in der Klinik für Psychiatrie und Psychotherapie hinsichtlich der rechtlichen Grundlagen und eventuell erforderlicher Maßregeln mit neuen Fragen konfrontiert. Der hier geschilderte Fall könnte deshalb als Beispiel für die Komplexität der Anwendung juristischer Normen im klinischen Alltag herangezogen werden, das auch wichtige Informationen für den Gesetzgeber hinsichtlich eventueller Unzulänglichkeiten bei der praktischen Umsetzung beinhalten dürfte.

Eine Verlegung des Patienten in eine psychiatrische Klinik auf freiwilliger Basis (zumal dort auch die somatische Diagnostik erfolgen konnte) war aufgrund der psychischen Störung (Suchterkrankung) durchaus die angemessene Wahl. Bei fehlender Zustimmung des Betroffenen wäre allerdings unbedingt zu prüfen gewesen, ob die Anwendung von Zwangsmaßnahmen aufgrund der psychischen Erkrankung durch Eigen- oder Fremdgefährdung gerechtfertigt gewesen wäre. Zur Auflösung und weiteren Einordung des Falles siehe: „Diskussion der Kasuistik im Kontext des Infektionsschutzes und der rechtlichen Rahmenbedingungen“.

\section{SARS-CoV-2-Pandemie}

Anlässlich der Situation im Zusammenhang mit der SARS-CoV-2-Infektionserkrankung müssen sich immer mehr Menschen in eine 14-tägige Quarantäne begeben. Vor Kurzem hat der Präsident des „Europäischen Ausschusses zur Verhütung von Folter und unmenschlicher oder erniedrigender Behandlung oder Stra$\mathrm{fe}^{\mathrm{e}}$ eine Grundsatzerklärung zur Behandlung von Personen im Freiheitsentzug veröffentlicht. Der Ausschuss erkennt darin die Notwendigkeit durchgreifender Maßnahmen bei einer SARS-CoV-2-Infektionserkrankung an, weist aber gleichzeitig auf die fortbestehende Pflicht hin, auch in dieser Situation Personen, denen die Freiheit entzogen worden ist, vor jeder Form unmenschlicher oder erniedrigender Behandlung zu schützen [1]. Nun stellt sich im klinischen Alltag der Psychiatrie die Frage, auf welcher Rechtsgrundlage Patienten, die aufgrund einer psychischen Erkrankung zu keiner freien Willensbildung fähig sind, bei einem zusätzlichen Verdacht auf eine SARS-CoV2-Infektion bzw. mit einer bestätigten COVID-19-Erkrankung versorgt werden schlechter führbar war, erfolgte nach 


\section{Infobox 1 Infektionsschutzge- setz $\S 30$}

Gesetz zur Verhütung und Bekämpfung von Infektionskrankheiten beim Menschen (Infektionsschutzgesetz, IfSG)

§ 30 Abs. 1, 2 Quarantäne

(1) Die zuständige Behörde hat anzuordnen, dass Personen, die an Lungenpest oder an von Mensch zu Mensch übertragbarem hämorrhagischem Fieber erkrankt oder dessen verdächtig sind, unverzüglich in einem Krankenhaus oder einer für diese Krankheiten geeigneten Einrichtung abgesondert werden. Bei sonstigen Kranken sowie Krankheitsverdächtigen, Ansteckungsverdächtigen und Ausscheidern kann angeordnet werden, dass sie in einem geeigneten Krankenhaus oder in sonst geeigneter Weise abgesondert werden, bei Ausscheidern jedoch nur, wenn sie andere Schutzmaßnahmen nicht befolgen, befolgen können oder befolgen würden und dadurch ihre Umgebung gefährden.

(2) Kommt der Betroffene den seine Absonderung betreffenden Anordnungen nicht nach oder ist nach seinem bisherigen Verhalten anzunehmen, dass er solchen Anordnungen nicht ausreichend Folge leisten wird, so ist er zwangsweise durch Unterbringung in einem abgeschlossenen Krankenhaus oder einem abgeschlossenen Teil eines Krankenhauses abzusondern. Ansteckungsverdächtige und Ausscheider können auch in einer anderen geeigneten abgeschlossenen Einrichtung abgesondert werden. Das Grundrecht der Freiheit der Person (Artikel 2 Abs. 2 Satz 2 Grundgesetz) kann insoweit eingeschränkt werden. Buch 7 des Gesetzes über das Verfahren in Familiensachen und in den Angelegenheiten der freiwilligen Gerichtsbarkeit gilt entsprechend.

sollen. Wenn bei Patienten mit einer psychischen Erkrankung keine freie Willensbildung möglich ist, ihr Wohl und das ihrer Umgebung aber akut und ernsthaft gefährdetsind, dann kann in seltenen Fällen die Anwendung von Zwangsmaßnahmen in Form freiheitsbeschränkender Maßnahmen oder einer Zwangsbehandlung durchaus gerechtfertigt und notwendig sein [2-4]. Möglicherweise sind Ärzte jedoch unsicher bezüglich derinfrage kommenden Rechtsgrundlagen von Zwangsmaßnahmen während der Corona-Pandemie: wann und unter welchen rechtlichen Voraussetzungen also solche Maßnahmen geboten sind.

In Deutschland können Unterbringungs- und andere Zwangsmaßnah- men auf der Basis länderspezifischer öffentlich-rechtlicher Vorschriften (Psychisch-Kranken-(Hilfe-)Gesetze (Psy$\operatorname{chK}(\mathrm{H}) \mathrm{G})$ ), im Rahmen des zivilrechtlichen Betreuungsrechts (BGB) oder schließlich aufgrund strafrechtlicher Regelungen (rechtfertigender Notstand, Maßregelvollzug, StGB) durchgeführt werden. Bislang wenig bekannt und beachtet waren die Regelungen des Infektionsschutzgesetzes (IfSG), die im Rahmen der SARS-CoV-2-Pandemie aber aktuelle Bedeutung erlangen (zur Übersicht s. • Abb. 1).

\section{Vorgehen bei Personen mit V.a. SARS-CoV-2-Infektion bzw. COVID-19}

Im Gesetz zur Verhütung und Bekämpfung von Infektionskrankheiten beim Menschen (Infektionsschutzgesetz IfSG) sind in $\$ 30$ Abs. 1 bis 7 die Bestimmungen zur Quarantäne festgelegt (s. DInfobox 1). Eine unverzügliche Absonderung (Quarantäne) in einem geeigneten Krankenhaus ist zwingend bei Personen mit Lungenpest oder hämorrhagischem Fieber anzuordnen. Grundsätzlich kann laut $\$ 30$ Abs. 1 Satz 2 IfSG aber auch bei anderen Krankheiten und Krankheitsverdachtsfällen durch die zuständige Behörde eine Absonderung angeordnet werden, somit auch bei V. a. Infektion mit SARS-CoV-2 bzw. mit COVID-19. Sollten sich die Patienten hierzu nicht in der Lage sehen bzw. nicht einsichtig zeigen, kann die Quarantäne nach $₫ 30$ Abs. 2 IfSG auch gegen den Willen der Betroffenen durchgesetzt werden.

Bei Verdacht auf eine SARS-CoV-2Infektion oder bei einem bestätigten COVID-19-Fall greift also an erster Stelle das IfSG - in der Auslegung der zuständigen Behörde. Dem liegt das rechtliche Prinzip zugrunde, wonach das spezielle Gesetz grundsätzlich dem Allgemeingesetz vorgeht (lex sepcialis derogat legi generali; $[5,6])$. Nach $\$ 30$ Abs. 1 IfSG kann bei Menschen, die an einer von Mensch zu Mensch übertragbaren Infektionserkrankung erkrankt oder dessen verdächtig sind, eine räumliche Isolierung angeordnet werden. Aufgrund der „Kann“-Formulierung im Gesetz sind hinsichtlich der Anwendung dieser Vorschrift bei COVID-19-Erkrankten oder SARS-CoV-2-Verdachtspersonen unterschiedliche Interpretationen und Vorgehensweisen zu erwarten. Regionale Unterschiede sind denkbar, z. B. unter Heranziehung regionaler oder lokaler Besonderheiten wie Bevölkerungsdichte oder Prävalenzzahlen, der Versorgungssituation oder der Lebensumstände der Betroffenen.

Falls eine Quarantäne angeordnet wird und der Betroffene diesen Anordnungen nicht nachkommt oder nachkommen kann, so kann er zwangsweise durch Unterbringung z. B. in einem abgeschlossenen Teil eines Krankenhauses abgesondert werden. Das Grundrecht der Freiheit der Person (Art. 2 Abs. 2 Satz 2 GG) kann hiermit eingeschränkt werden, vgl. $\$ 30$ Abb. 3 IfSG. Die Anordnung einer Quarantäne gemäß $₫ 30$ Abs. 1 IfSG erfolgt durch die zuständige Gesundheitsbehörde (Gesundheitsamt), die auch über die im Einzelfall zu treffenden Maßnahmen entscheidet. Das konkrete Vorgehen nach IfSG ist dabei im klinischen Alltag bislang einerseits wenig bekannt, andererseits sind entsprechende Verfahrensregeln in der Praxis meist nicht ad hoc verfügbar, zumal es kaum veröffentlichte Rechtsprechung ordentlicher Gerichte oder Literatur zu dieser Thematik gibt.

\section{Vorgehen bei Personen mit einer psychischen Erkrankung und mit V.a. SARS-CoV-2-Infektion bzw. COVID-19}

Anders verhält es sich bei Personen mit psychischen Störungen. Hier regeln die PsychKG der Länder das Vorgehen bei akuter Eigen- und/oder Fremdgefährdung mit einem meist routiniert eingespielten Handlungsablauf zwischen Kliniken, Behörden und Betreuungsgerichten. Ist ein Patient aufgrund seiner psychischen Erkrankung nicht zur freien Willensbildung fähig und besteht eine Eigen- und/oder Fremdgefährdung, dann kann eine Unterbringung nach PsychKG maßgeblich werden. Laut Einschätzung des Bayerischen Justizministeriums ist der Anwendungsbereich des PsychKG neben dem IfSG eröffnet, da beide Ge- 
setze eine Fremdgefährdung vermeiden wollen. Zwar sind die im Vordergrund stehenden Ursachen der Fremdgefährdung unterschiedlich (IfSG: Ansteckung mit infektiöser, gefährlicher Krankheit, PsychKG: psychische Störung), ist jedoch eine mit SARS-CoV-2-infizierte, krankheits- oder ansteckungsverdächtige Person aufgrund einer psychischen Störung nicht in der Lage, Anweisungen des Gesundheitsamts so zu befolgen, dass für Dritte keine Gefahr ausgeht, kann eine öffentlich-rechtliche Unterbringung in Betracht gezogen werden. Im Rahmen einer öffentlich-rechtlichen Unterbringung besteht in der Regel eine Rechtsgrundlage auch für ggf. zusätzliche erforderliche Sicherungsmaßnahmen. Bei einer fehlenden freien Willensbildung oder erheblich beeinträchtigter Einsichts- oder Steuerungsfähigkeit aufgrund einer psychischen Erkrankung können also die Psych(H)KG der Länder zur Anwendung kommen (in Bayern abhängig davon, ob die Tatbestandsvoraussetzungen des Art. 5 BayPsychKHG erfüllt sind, s. - Infobox 2). Geht von Patienten aufgrund einer psychischen Erkrankung mit eingeschränkter freien Willensbildung eine akute Eigen- oder Fremdgefährdung aus, regeln also die PsychKG der einzelnen Bundesländer, wie eine Unterbringung und eventuell weitere Zwangsmaßnahmen, z. B. eine Fixierung oder eine Zwangsbehandlung, einzuleiten sind. Bei den PsychKG ist neben dem Schutz der Betroffenen vor Eigengefährdung im Gegensatz zum Betreuungsrecht auch der Schutz des öffentlichen Interesses von Belang.

In Bayern sind die Eingangsvoraussetzungen einer Unterbringung in Artikel 5 BayPsychKHG geregelt:

Eine SARS-CoV-2-Infektion bzw. COVID-19 kann nach Einschätzung des Amts für öffentlich-rechtliche Unterbringung (AfoerU, Nördlingen, in Bayern zuständige Aufsichtsbehörde für die öffentlich-rechtlichen Unterbringung nach BayPsychKHG) im Einzelfall eine potenzielle Fremdgefährdung darstellen, d.h. für Personen, die aufgrund einer psychischen Erkrankung eine erhebliche Beeinträchtigung der Einsichtsund Steuerungsfähigkeit aufweisen (Tatbestandsvoraussetzung: Art. 5 BayPsy-
chKHG), kann eine Unterbringung nach BayPsychKHG beantragt und angeordnet werden, um den Schutz des Lebens und der Gesundheit anderer zu gewährleisten. Hinsichtlich der Einschätzung einer Fremdgefährdung ist hierbei der Grundsatz der Verhältnismäßigkeit zu beachten.

Sollte also bei infizierten Personen oder Verdachtsfällen eine räumliche Isolierung (Quarantäne i. e.S.) im Rahmen des IfSG nicht ausreichen und zusätzliche Zwangsmaßnahmen (z.B. unmittelbare mechanische Beschränkung) zum Schutz der Allgemeinheit notwendig sein, muss erwogen werden, ob die Einsichtsfähigkeit nicht durch eine psychische Erkrankung eingeschränkt ist. In solchen Fällen wären entsprechende Maßnahmen nach eingehender psychiatrischer Prüfung im Rahmen der länderspezifischen PsychKG oder Unterbringungsgesetze zu beantragen. Das Vorgehen erfolgt nach BayPsychKHG, wie in Teil 2 „öffentlich-rechtliche Unterbringung" vorgesehen: Maßgeblich für die sofortige vorläufige Unterbringung sind Art. 11, 12 und 13 mit den Verfahrenshinweisen in Art. 14 BayPsychKHG.

Allerdings ist zu beachten, dass auch eine öffentlich-rechtliche Unterbringung (z. B. in Bayern unter Voraussetzung des Art. 5 BayPsychKHG) nur angeordnet werden darf, wenn die Verhältnismäßigkeit gewahrt bleibt. So ist bei Personen, die aufgrund einer psychischen Störung zwar in ihrer Einsichts- und Steuerungsfähigkeit erheblich beeinträchtigt sind, die aber die Quarantänebestimmungen des Gesundheitsamts einhalten, eine Unterbringung nach PsychKG nicht angezeigt. Bei Personen ohne eingeschränkte Einsichts- oder Steuerungsfähigkeit ist eine Unterbringung nach PsychKHG keinesfalls möglich. In diesen Fällen erfolgt eine Anordnung der Quarantäne nach $₫ 30$, Infektionsschutzgesetz. Hierfür zuständig sind die Gesundheitsämter. Bedarf es einer zwangsweisen Absonderung, kann diese über die Gesundheitsämter richterlich angeordnet werden.

In Bayern ist in solchen Fällen über die Rufnummer 112 der Infektiologische Rufdienst (Erreichbarkeit 24/7) zu kontaktieren; diese Rufbereitschaft ist im Gesundheitsamt angesiedelt. Es kann dort
Nervenarzt 2021 · 92:501-506

https://doi.org/10.1007/s00115-020-01002-y

(c) Springer Medizin Verlag GmbH, ein Teil

von Springer Nature 2020

\author{
K. Adorjan · D. Gaudernack · J. Beer - \\ C. von Massenbach · P. Falkai . \\ T. Pollmächer $\cdot 0$. Pogarell
}

\section{Zwangsmaßnahmen und die SARS-CoV-2-Pandemie}

\section{Zusammenfassung}

Im klinischen Alltag der Psychiatrie stellt sich regelmäßig die Frage, unter welcher Rechtsgrundlage Patienten, die aufgrund einer psychischen Erkrankung zu keiner freien Willensbildung fähig sind, bei einem zusätzlichen Verdacht auf eine SARS-CoV-2Infektion bzw. mit einer bestätigten Covid19-Erkrankung versorgt werden sollen. Womöglich sind Ärzte unsicher bezüglich der in Frage kommenden Rechtsgrundlagen von Zwangsmaßnahmen während der Corona-Pandemie: Wann und unter welchen rechtlichen Voraussetzungen also solche Maßnahmen geboten sind. Im vorliegenden Artikel werden die hierfür aktuell geltende rechtliche Regelungen in Deutschland vorgestellt und diskutiert.

Schlüsselwörter

Zwangsmaßnahmen · SARS-CoV-2 . Pandemie

\section{Coercive measures and the SARS-CoV-2 pandemic}

\section{Abstract}

In the clinical daily work of psychiatry, the question regularly arises under which legal conditions patients who are not able to form free will due to a mental illness should be treated in case of an additional suspicion of a SARS-CoV-2 infection or with a confirmed Covid-19 disease. However, physicians may be uncertain about the legal basis for coercive measures during the corona pandemic: when and under which legal conditions such measures are required. This article presents and discusses in detail the legal regulations currently applicable in Germany.

Keywords

Coercive measures $\cdot$ SARS-CoV-2 $\cdot$ Pandemic 


\section{Infobox 2 Artikel 5 BayPsychKHG}

Art. 5 Voraussetzungen der Unterbringung,

Verhältnismäßigkeitsgrundsatz

(1) ${ }^{1}$ Wer auf Grund einer psychischen

Störung, insbesondere Erkrankung, sich

selbst, Rechtsgüter anderer oder das

Allgemeinwohl erheblich gefährdet, kann

ohne oder gegen seinen Willen untergebracht werden, es sei denn seine Einsichts- und

Steuerungsfähigkeit ist nicht erheblich

beeinträchtigt. ${ }^{2}$ Für eine Unterbringung nach diesem Gesetz anstelle einer Unterbringung

nach den Vorschriften des Bürgerlichen

Gesetzbuches (BGB) kann in Fällen der

Selbstgefährdung insbesondere sprechen,

dass die Unterbringung voraussichtlich nicht

länger als sechs Wochen dauern wird und

keine Betreuung und keine ausreichende

Vorsorgevollmacht besteht. ${ }^{3}$ Bei Kindern und

Jugendlichen ist eine Unterbringung nach

$\S 1631 \mathrm{~b}$ BGB vorrangig.

(2) ${ }^{1}$ Die Unterbringung darf nur angeordnet werden, wenn die Gefährdung nicht durch weniger einschneidende Mittel abgewendet werden kann, insbesondere auch nicht durch die Hinzuziehung eines Krisendienstes und durch Hinzuziehung der oder des gesetzlichen Vertreters. ${ }^{2}$ Sie darf nicht zu einem Nachteil führen, der zu dem angestrebten Erfolg erkennbar außer Verhältnis steht. ${ }^{3} \mathrm{Sie}$ ist nur solange zulässig, bis ihr Zweck erreicht ist oder nicht mehr erreicht werden kann.

${ }^{4}$ Die Sätze 1 bis 3 gelten entsprechend für Maßnahmen während der Unterbringung. ${ }^{5}$ Von mehreren möglichen und geeigneten Maßnahmen ist diejenige zu wählen, die die untergebrachte Person voraussichtlich am wenigsten beeinträchtigt.

der Sachverhalt geschildert und das weitere Vorgehen erörtert werden.

Über das Gesundheitsamt erfolgt ein Quarantänebeschluss:

1. im SARS-CoV-2-Verdachtsfall bis zum Vorliegen der Befunde (Bestätigung des Verdachts oder Ausschluss),

2. bei bestätigtem Nachweis einer

Infektion für einen Zeitraum von mindestens 14 Tagen.

\section{Sonderfall - Rechtfertigender Notstand ( $\$ 34$ StGB)}

Abgesehen von der räumlichen Isolierung ist die Anwendung weiterer freiheitsentziehender Zwangsmaßnahmen allein aufgrund des IfSG nicht gestattet. Nach Einschätzung des bayerischen Justizministeriums sind bei einer Fixierung die Grundsätze der Entscheidung des BVerfG zur Fixierung in der öffentlich-rechtlichen Unterbringung vom

\section{Infobox 3 Rechtfertigender Notstand (§ 34 StGB)}

Ein rechtfertigender Notstand kommt nur in Notfällen zum Einsatz: Auf der Basis eines rechtfertigenden Notstandes können Zwangsmaßnahmen bei vitaler Bedrohung zum Schutz der Allgemeinheit und zum

Eigenschutz unverzüglich durchgeführt werden; die normative Grundlage für derartige Maßnahmen findet sich im § 34 des Strafgesetzbuches. Sollte eine Person also z. B. aggressiv reagieren und dabei sich und andere gefährden, könnte diese Vorschrift angewandt werden.

24.07.2018 zu beachten. Das IfSG erlaubt an freiheitsentziehenden Maßnahmen ausdrücklich nur die zwangsweise Absonderung. Somit bietet das IfSG keine Rechtsgrundlage für mechanische Beschränkungen im Sinne des BVerfG, für die zudem ein richterlicher Anordnungs- und Genehmigungsvorbehalt besteht. Sollte bei Personen, die nach IfSG abgesondert wurden, aufgrund besonderer Umstände zusätzlich eine Fixierung erforderlich werden, kommt als Rechtfertigungsgrundlage lediglich $\S 34$ StGB (rechtfertigender Notstand) infrage ([7]; s. ๑ Infobox 3).

\section{Räumliche Gegebenheiten}

Nach $₫ 30$ Abs. 2 IfSG sind Absonderungsmaßnahmen, die zwangsweise durch Unterbringung durchgeführt werden müssen, in einem abgeschlossenen Krankenhaus oder einem abgeschlossenen Teil eines Krankenhauses durchzuführen. Die notwendigen Räume und Einrichtungen sind von den Ländern zu schaffen und zu unterhalten $(\$ 30$ Abs. 6 IfSG). Hierfür kommen nicht psychiatrische Kliniken infrage, sondern andere Einrichtungen der medizinischen Versorgung, und gerade Menschen, die primär an einer körperlichen Erkrankung leiden, sollten ausschließlich in somatischen Kliniken untergebracht werden. Insbesondere ist eine gesellschaftliche oder staatliche Instrumentalisierung psychiatrischer Kliniken für Menschen, die eine häusliche Quarantäne verweigern, unbedingt $\mathrm{zu}$ vermeiden. Aber auch Menschen mit psychischen Erkrankungen, die ernsthafte körperliche
Symptome aufweisen, sollten bevorzugt in somatischen Kliniken behandelt werden (vgl. BayPsychKG). In der klinischen Praxis könnte es jedoch insofern zu Schwierigkeiten kommen, als die Gesetzgebung die Unterbringung von Patienten bei einer fehlenden freien Willensbildung oder erheblich beeinträchtigter Einsichts- oder Steuerungsfähigkeit auf geschlossenen Station erlaubt, somatische Kliniken aber über derartigen Einrichtungen oft nicht verfügen. Folglich sollten Versorgungsstrukturen entsprechend ausgebaut werden (z.B. durch Bereitstellung von Überwachungsräumen in somatischen Kliniken).

\section{Fazit}

In den vergangenen Jahrzehnten haben juristische Normen die Patientenautonomie erheblich gestärkt. So sind in Art. 1 (Menschenwürde), in Art. 2 (Freiheit) und in Art. 3 (Gleichheit) des Grundgesetzes der Bundesrepublik Deutschland die Grundrechte des Menschen als fundamentale Leitprinzipien festgeschrieben. Auch die UN-Behindertenrechtskonvention von 2006 (UNBRK) betont in Art. 12 das Selbstbestimmungsrecht von Patienten. Eine Reihe von Entscheidungen des Bundesverfassungsgerichtes (BVerfG) seit 2011 stärkte ebenfalls weiter die Patientenautonomie. In Zeiten einer Pandemie könnten allerdings Situationen entstehen, in denen die Patientenautonomie und der Schutz der Allgemeinheit in Konflikt geraten und nach Abwägung aller Umstände dem Schutz der Allgemeinheit Vorrang eingeräumt werden muss. Ärztliches Handeln orientiert sich primär am Wohl der Patienten, weshalb rein fremdnütziges medizinisches Handeln, z. B. zum Schutz der Allgemeinheit, insbesondere dann problematisch ist, wenn Menschen mit psychischen Erkrankungen ausschließlich unter vorsorglichen $\mathrm{Si}$ cherungsaspekten untergebracht sind. Hier sind die Rechtfertigungsgründe besonders streng abzuwägen und hinsichtlich der Verhältnismäßigkeit zu prüfen [8]. 


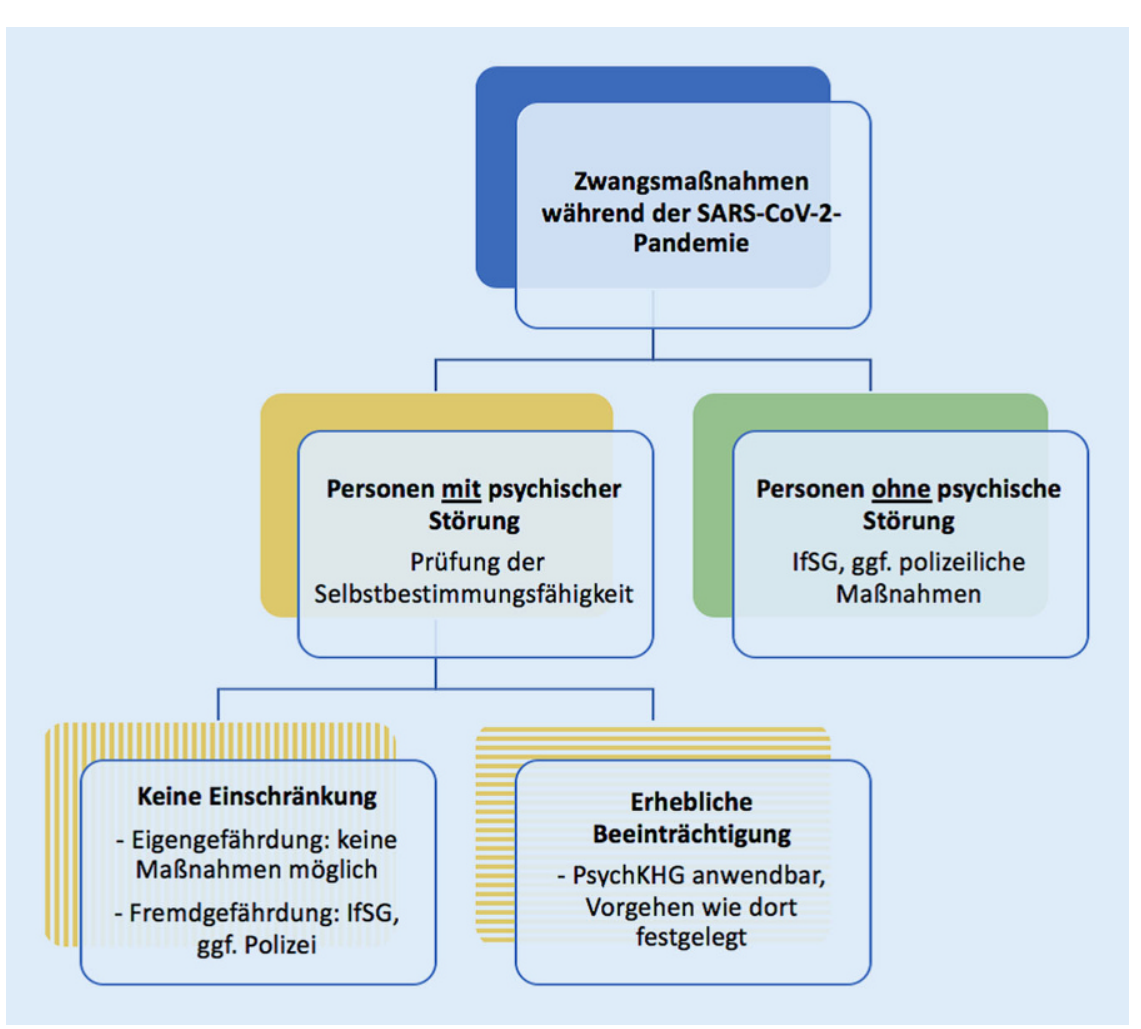

Abb. $1 \Delta$ Algorithmus:Zwangsmaßnahmen und die SARS-CoV-2-Pandemie.IfSG Infektionsschutzgesetz, PsychKHG Psychisch-Kranken-Hilfe-Gesetze

\section{Diskussion der Kasuistik im Kontext des Infektions- schutzes und der rechtlichen Rahmenbedingungen}

Die Unterbringung eines Menschen in einer psychiatrischen Klinik ist gegen seinen natürlichen Willen grundsätzlich nur dann gerechtfertigt, wenn (1) eine Eigenund/oder Fremdgefährdung vorliegt, die auf eine psychische Erkrankung zurückzuführen ist, und (2) die freie Willensbildung aufgrund der psychischen Störung beeinträchtigt ist.

\section{Lag eine Eigengefährdung des Betroffenen vor?}

Im konkreten Fall war der Patient mit 2,5\%o BAK alkoholisiert, initial zeitlich und örtlich unscharf orientiert und die Einsichtsfähigkeit war beeinträchtigt.

a) Intoxikation und Alkoholabhängigkeit

Hier ist anzumerken, dass eine Eigengefährdung nicht von der Höhe labormedizinisch gemessener Alkoholwerte abgeleitet werden kann, sondern von den jeweiligen psychopathologischen Befunden. Es zeigte sich hier psychopathologisch eine Orientierungsstörung, die im Rahmen der Fürsorgepflicht durchaus einen Grund darstellte, den Patienten zum Verbleiben auf der Station zu bewegen. Eine unmittelbare Eigengefährdung war jedoch aufgrund der psychischen Störung (Alkoholintoxikation) in Folge mangelnder Einsichtsfähigkeit und Compliance sowie durch mögliche Unfälle und durch einen potenziellen komplizierten Alkoholentzug (mit Delir oder entzugsbedingten Krampfanfällen) in Betracht zu ziehen.

b) Infektionserkrankung (COVID-19) Zudem bestand zu diesem Zeitpunkt klinisch, radiologisch und labormedizinisch ein hochgradiger COVID19-Verdacht. Eine unbehandelte COVID-19-Erkrankung stellt eine prädiktiv kaum abschätzbare Gefährdung des Betroffenen dar. Angesichts des hochgradigen COVID-19-Verdachts leitete sich auch aus dieser Konstellation eine Eigengefährdung $\mathrm{ab}$, die zumindest eine weitere $\mathrm{Di}$ agnostik (SARS-CoV-2-Test) und Verlaufsbeobachtung rechtfertigte.

Zu a + b. Ohne erhebliche Beeinträchtigung der Selbstbestimmungsfähigkeit ist eine Unterbringung wegen Eigengefährdung in Folge der Alkoholerkrankung (Intoxikation) gegen den natürlichen Willen des Patienten nicht erlaubt; ebenso wenig beim Vorliegen einer potenziell schwerwiegenden Infektionserkrankung. Im konkreten Fall lag nach Einschätzung des Konsiliarius aufgrund der Alkoholintoxikation eine akute Eigengefährdung vor.

\section{Lag eine Fremdgefährdung vor und wie wäre diese zu bewerten?}

Da ein Verdacht auf eine SARS-CoV2-Infektion bestand und der Patient nicht ausreichend kooperierte, war eine Fremdgefährdung in Betracht $\mathrm{zu}$ ziehen. Bei SARS-CoV-2 handelt es sich um einen hochansteckenden Erreger, der im Falle einer Übertragung zu potenziell lebensbedrohlichen Erkrankungen Dritter führen kann. Zwangsmaßnahmen bei Fremdgefährdung im Kontext begleitender psychischer Störungen erfordern jedoch eine besonders strenge Kausalitätsprüfung:

a) Eine Fremdgefährdung durch SARSCoV-2 durch die Weigerung eines

Menschen, ärztlichen Anweisungen

Folge zu leisten, stellt zunächst keine psychische Störung und somit keine Grundlage einer psychiatrischen Unterbringung gegen den Willen des Patienten dar.

b) Auch beim Vorliegen einer psychischen Störung erlaubt eine Fremdgefährdung aufgrund der Infektionserkrankung eine Unterbringung gegen den natürlichen Willen des Patienten in einer psychiatrischen Klinik nicht, sofern die Eingangsvoraussetzungen (erhebliche Beeinträchtigung der Einsichtsfähigkeit aufgrund der psychischen Störung) nicht zweifelsfrei vorliegen.

c) Wenn jedoch eine Person, die mit dem Corona-Virus infiziert, krankheits- oder ansteckungsverdächtig ist und aufgrund einer psychischen 
Störung nicht in der Lage ist, den Anweisungen des Gesundheitsamts so $\mathrm{zu}$ folgen, dass für Dritte keine Gefahr von ihr ausgeht, so kann sie laut IfSG und BayPsychKHG gegen ihren natürlichen Willen in einer psychiatrischen Klinik untergebracht werden.

Im konkreten Fall bestand eine Fremdgefährdung aufgrund der psychischen Erkrankung durch mangelnde Kooperation, um z. B. Schutzmaßnahmen zu befolgen, bei gelichzeitig vorliegendem Verdacht auf eine hochgradig ansteckende Infektionserkrankung. Diese Konstellation entspricht Punkt c (siehe oben).

\section{Räumliche Versorgungsoptionen}

Laut IfSG sollen Patienten mit Verdacht auf eine SARS-CoV-2-Infektion in einem abgeschlossenen Teil eines Krankenhauses abgesondert untergebracht werden.

Im konkreten Fall konnte der Betroffene in einer separaten, hierfür eingerichteten COVID-Station der Psychiatrischen Klinik zur Behandlung der psychischen Erkrankung (Intoxikationsund Entzugsbehandlung) und zugleich Vervollständigung der somatischen Diagnostik übernommen werden. Angesichts der Gesamtkonstellation konnte die Aufnahme in der Psychiatrischen Klinik 1. zum Schutz des Betroffenen (Fürsorgepflicht) und 2. unter Berücksichtigung der Infektionsschutzmaßnahmen gerechtfertigt werden. Der Patient blieb letztlich freiwillig zur Behandlung. Auch wenn die Voraussetzungen für eine Unterbringung gegen den natürlichen Willen des Patienten erfüllt gewesen wären, mussten diese Maßnahmen nicht angewendet werden.

Liegt keine psychische Erkrankung vor, sollten Personen mit V. a. eine SARSCoV-2-Infektion nicht in einem psychiatrischen Krankenhaus untergebracht werden; eben so wenig, wenn bei stabilem psychopathologischem Befund die somatische Komponente eindeutig im Vordergrund steht. Eine unzulässige Instrumentalisierung des psychiatrischen Versorgungssystems sollte unbedingt vermieden werden.

\section{Korrespondenzadresse}

\section{Kristina Adorjan}

Klinik für Psychiatrie und Psychotherapie, LMUKlinikum

Nußbaumstr. 7, 80336 München, Deutschland Kristina.Adorjan@med.uni-muenchen.de

\section{Einhaltung ethischer Richtlinien}

Interessenkonflikt. K. Adorjan, D. Gaudernack, J. Beer, C. von Massenbach, P. Falkai, T. Pollmächer und $\mathrm{O}$. Pogarell geben an, dass kein Interessenkonflikt besteht.

Für diesen Beitrag wurden von den Autoren keine Studien an Menschen oder Tieren durchgeführt. Für die aufgeführten Studien gelten die jeweils dort angegebenen ethischen Richtlinien.

\section{Literatur}

1. Europäisches Komitee zur Verhütung von Folter und unmenschlicher oder erniedrigender Behandlung oder Strafe (CPT): Europäisches Komitee zur Verhütung von Folter und unmenschlicher oder erniedrigender Behandlung oder Strafe (CPT): Grundsatzerklärung zur Behandlung von Personen im Freiheitsentzug im Zusammenhang mit derCoronavirus (COVID-19)-Pandemie. https://rm. coe.int/16809cfde3.Zugegriffen: 20.08.2020

2. Steinert T et al (2014) Zwangsmaßnahmen in psychiatrischen Kliniken in Deutschland. Gegenwärtige Praxis (2012). Nervenarzt 85:621-629

3. Maier W et al (2014) Achtung der Selbstbestimmung und Anwendung von Zwang bei der Behandlung von psychisch erkrankten Menschen. Eine ethische Stellungnahme der DGPPN. http:// www.dgppn.de/en/presse/pressemitteilungen/ detailansicht/article/307/achtung-der.html. Zugegriffen: 20.08.2020

4. PollmächerT (2015) Moral oder Doppelmoral? Das Berufsethos des Psychiaters im Spannungsfeld zwischen Selbstbestimmung, Rechten Dritter und Zwangsbehandlung. Nervenarzt 86(9):1148-1156

5. Bydlinski F (1991) Juristische Methodenlehre und Rechtsbegriff, 2. Aufl., S465

6. Larenz K (1991) Methodenlehre der Rechtswissenschaft, 6. Aufl., S267

7. JMS des Bayerischen Staatsministeriums der Justiz vom 1. April 2020,D2a-3475-I-3199/2020.

8. PollmächerT(2019) Zur Legitimität fremdnützigen Handelns in der Medizin und speziell in der Psychiatrie. Forens Psychiatr Psychol Kriminol. https://doi.org/10.1007/s11757-018-00517-0
Lockdown trifft berufstätige Frauen stärker als Männer

Ein Forschungsteam unter Prof. Dr. Bertolt Meyer, Inhaber der Professur Organisations- und Wirtschaftspsychologie an der Technischen Universität Chemnitz, hat die psychischen Belastungen infolge der Covid-19-Pandemie untersucht.

Dafür wurden im Zeitraum April bis Juni 2020 in drei Befragungswellen insgesamt 3.862 Personen befragt. Abgefragt wurden gezielt persönliche Faktoren wie Geschlecht, Familien- und Paarsituation sowie Arbeit im Homeoffice. Das Forschungsteam wollte herausfinden, ob es Zusammenhänge zwischen diesen persönlichen Faktoren und der Emotionalen Erschöpfung gibt und wie sich diese gegebenenfalls im Verlauf der Pandemie entwickeln.

Eines der Ergebnisse war, dass insbesondere die psychische Gesundheit der Frauen stärker angegriffen war als die der Männer. Wesentliche Faktoren waren das Vorhandensein von Vorschul- und Schulkindern im Haushalt in Kombination mit geschlossenen Schulen und Kitas sowie Arbeit im Homeoffice.

Die Forscher schlussfolgern daraus, dass die Pandemie insbesondere für Frauen mit stärkeren psychischen Belastungen verbunden ist, auch traditionelle Geschlechterrollen könnten ebenso verstärkt werden. Die Empfehlung an Regierungen und politische Entscheidungsträgerlnnen lautet daher, besonders die Milderung der psychischen Folgen bei Frauen durch die Pandemie im Blick zu haben.

Originalpublikation: Meyer, B., Zill, A., Dilba, D., Gerlach, R., \& Schumann, S. (2021). Employee psychological well-being during the COVID-19 pandemic in Germany: A longitudinal study of demands, resources, and exhaustion. International Journal of Psychology. Advance online publication. http://doi.org/10.1002/ijop.12743

Quelle: Technische Universität Chemnitz (www.tu-chemnitz.de) 